$\mathrm{C}_{42} \mathrm{H}_{30} \mathrm{~S}_{6}$ Mo (Schmp. $>360^{\circ}$ Zers.)

$\begin{array}{lllll}\text { Ber. C } 61,3 & \text { H } & 3,7 & \text { S } & 23,4\end{array}$

Mol.-Gew. 823,1;

Gef. C 61,0 H 3,8 S 23,3,

Mol.-Gew. 763.

$\mathrm{C}_{42} \mathrm{H}_{30} \mathrm{~S}_{6} \mathrm{~W}$ (Schmp. $>360^{\circ}$ Zers.)

Ber. C 55,4 H 3,3,

Gef. C 55,6 H 3,4.

Die drei Komplexe sind auf Grund von Pulveraufnahmen isotyp.

5 A. Davison, N. Edelstein, R. H. Holm u. A. H. Maki, J. Amer. chem. Soc. 86, 2799 [1964].
Analoge Komplexe dieser Metalle bilden sich auch bei der Reaktion der Carbonyle mit $\mathrm{CF}_{3} \mathrm{C}_{2} \mathrm{~S}_{2} \mathrm{CF}_{3}{ }^{5,6}$. Unsere Vermutung, daß es sich bei den vorliegenden Verbindungen um Planarkomplexe dieser Metalle handelt, wird somit hinfällig, wenngleich auf Grund der Ähnlichkeit der IR- und UV-Spektren auf eine dem Nickelkomplex nahe verwandte Elektronenstruktur geschlossen werden muß. Zur Problematik der Bindungsverhältnisse werden wir an anderer Stelle noch Stellung nehmen.

${ }^{6}$ R. B. KING, Inorg. Chem. 2, 641 [1963].

\section{Ein Beitrag zum Problem des Überganges des Triphenylchrom(III)-tristetrahydrofuranats in Aromaten-chrom(O)-Komplexe}

Von J. HäHLE und G. Stolze

Institut für Anorganische Chemie der Universität Jena und Forschungsstelle für Komplexchemie der Deutschen Akademie der Wissenschaften zu Berlin in Jena

(Z. Naturforschg. 19 b, 1081-1082 [1964] ; eingeg. am 14. August 1964)

Von Zeiss und Herwig ${ }^{1}$ wurde gefunden, daß das Triphenylchrom (III) -tristetrahydrofuranat beim Entfernen der Solvatmoleküle ein schwarzes Produkt liefert, welches bei der Hydrolyse Dibenzolchrom(O) und Benzol-diphenylchrom $(\mathrm{O})$ ergibt.

Eigene Untersuchungen zeigten, daß die Umwandlungsgeschwindigkeit des Triphenylchroms in das schwarze Produkt sehr von seiner Reinheit abhängt. Behandelt man aus $\mathrm{THF}^{*}$ umkristallisiertes $\mathrm{Cr}\left(\mathrm{C}_{6} \mathrm{H}_{5}\right)_{3} \cdot 3 \mathrm{THF}$ in geeigneter Weise mit n-Heptan, Benzol, Diäthyläther oder läßt man einen langsamen Argonstrom über das Präparat streichen, so erhält man grüne, luft- und feuchtigkeitsempfindliche Kristalle. Die quantitative Analyse ergab die Zusammensetzung $\mathrm{Cr}\left(\mathrm{C}_{6} \mathrm{H}_{5}\right)_{3} \cdot 2$ THF. Die Reaktion läuft also nach folgender Gleichung $a b$ :

$$
\mathrm{Cr}\left(\mathrm{C}_{6} \mathrm{H}_{5}\right)_{3} \cdot 3 \mathrm{THF} \rightleftarrows \mathrm{Cr}\left(\mathrm{C}_{6} \mathrm{H}_{5}\right)_{3} \cdot 2 \mathrm{THF}+\mathrm{THF} .
$$

Die Formulierung als $\sigma$-Phenylchromverbindung ergibt sich aus dem chemischen Verhalten und den physikalischen Eigenschaften. Durch Umsetzung mit $\mathrm{HgCl}_{2}$ werden 3 Mole Phenylquecksilberchlorid (Schmp. $250{ }^{\circ} \mathrm{C}$ ) pro Mol Chrom erhalten. Bei der Hydrolyse entstehen Benzol und $\operatorname{Cr}(\mathrm{OH})_{3}$. Das Tetrahydrofuran wurde durch thermischen Abbau quantitativ erfaßt und durch Siedepunktsbestimmung nach $\mathrm{Siwoloboff}$ (Sdp. $\left.66-67{ }^{\circ} \mathrm{C}\right)$ charakterisiert. Magnetische Messungen ergaben 3,98 B.M. entsprechend 3 ungepaarten Elektronen am Chrom. Mit THF entsteht sofort wieder das Tristetrahydrofuranat. In Benzol und Dioxan löst sich die Verbindung, jedoch sind die Lösungen nicht lange haltbar.

1 W. Herwig u. H. H. Zeiss, J. Amer. chem. Soc. 80, 6561 [1957] ; W. Herwig u. H. H. Zeiss, J. Amer. chem. Soc. 81, 4798 [1958].
Die Isolierung und Charakterisierung dieser Substanz erklärt die schon öfter beobachteten Grünfärbungen einiger anderer Triaryl-chrom-tristetrahydrofuranate ${ }^{2}$.

Das grüne Bistetrahydrofuranat ist nicht unbegrenzt haltbar. Es wandelt sich im trockenen Zustand im Verlaufe von Tagen bis Wochen in ein schwarzes Produkt um. Verfolgt man dabei das magnetische Verhalten, so ist eine Abnahme der magnetischen Suszeptibilität zu erkennen:

\begin{tabular}{|c|c|c|}
\hline $\begin{array}{c}\text { Tage nach } \\
\text { der } \\
\text { Präparation }\end{array}$ & $\begin{array}{c}\chi g \cdot 10^{-6} \\
{\left[\mathrm{~cm}^{3} \cdot \mathrm{g}^{-1}\right]}\end{array}$ & $\begin{array}{c}\text { Temp. } \\
{\left[{ }^{\circ} \mathrm{K}\right]}\end{array}$ \\
\hline 2 & $14,98=3,97$ B.M. & 290,8 \\
30 & 9,55 & 290,9 \\
44 & 8,51 & 291,7 \\
64 & 6,80 & 291,3 \\
155 & 5,35 & 290,9 \\
\hline
\end{tabular}

Tab. 1. Abnahme der magnetischen Suszeptibilität von $\mathrm{Cr}\left(\mathrm{C}_{6} \mathrm{H}_{5}\right)_{3} \cdot 2 \mathrm{THF}$ in Abhängigkeit von der Zeit.

In Berührung mit Benzol oder Diäthyläther erfolgt die Umwandlung innerhalb weniger Stunden. Es entsteht ein schwarzer Bodenkörper, während die überstehende Lösung eine rotorange bis dunkelbraune Farbe besitzt. Durch Extraktion mit geeigneten organischen Lösungsmitteln wie Hexan oder auch Diäthyläther können lösliche Anteile von dem schwarzen Bodenkörper ohne vorherige Hydrolyse abgetrennt werden. Die weitere Untersuchung ergibt, daß die Lösung beträchtliche Mengen Diphenyl (Schmp. 67-68 ${ }^{\circ} \mathrm{C}$; Mischschmelzpunkt ohne Depression; IR-Spektrum) und Bis-diphenylchrom $(\mathrm{O})$ enthält.

Die Identifizierung des Aromatenkomplexes als Bisdiphenylchrom $(\mathrm{O})$ erfolgte

1. durch die quantitative Jodierung zum Bis-diphenylchrom(I)-jodid und dessen papierchromatographische Charakterisierung,

* THF $=$ Tetrahydrofuran.

2 F. Hein, J. Hähle u. G. Stolze, Mber. dtsch. Akad. Wiss. Berlin 5, 528 [1963]. 
2. durch Vergleich des IR-Spektrums einer vom Diphenyl befreiten Hexanlösung mit der Lösung einer nach der reduzierenden Friedel-Craf $\mathrm{t}$ s-Methode hergestellten Probe ${ }^{3}$,

3. durch die Farbreaktion mit Chloranil ${ }^{4}$.

Die Abtrennung des Bis-diphenylchrom $(\mathrm{O})$ vom schwarzen Bodenkörper vor der Hydrolyse erklärt den Befund von Zeiss und Herwig, daß bei der Deuterolyse des Reaktionsproduktes von ätherischem Phenylmagnesiumbromid und $\mathrm{CrCl}_{3}$ im Bis-diphenylchrom kein Deuterium zu finden ist, da dieses schon vor der Deuterolyse entsteht ${ }^{5}$.

Im Gegensatz zu Zeiss, Herwig und Tsutsur ${ }^{1,}{ }^{6}$, die als Hauptprodukte des Überganges vom Triphenylchrom in die Aromatenkomplexe neben Spuren Bis-diphenylchrom nur Dibenzolchrom und Benzoldiphenylchrom im Molverhältnis 1 : 1 angeben, haben wir unter unseren Bedingungen gefunden, daß das Bis-diphenylchrom $(0)$ in Ausbeuten von über $10 \%$ der eingesetzten Chrommenge entsteht.

Erst bei der Hydrolyse des verbleibenden pyrophoren schwarzen Rückstandes entstehen Dibenzolchrom $(\mathrm{O})$, Benzol-diphenylchrom $(\mathrm{O})$, Chromhydroxid, Wasserstoff und Benzol. Der Wasserstoff, der durch die qualitative Molybdänblaureaktion und durch quantitative Bestimmung in der Dennis-Pipette als solcher erkannt wurde, entstammt einem noch nicht aufgeklärten Redoxvorgang, da bei der Deuterolyse praktisch reines $D_{2}$ entsteht (Isotopenhäufigkeit 93\% D*). Das Benzol wurde im ätherischen Extrakt des Hydrolysates IR-spektroskopisch durch das Auftreten der intensivsten Bande bei $677 \mathrm{~cm}^{-1}$ nachgewiesen.

Quantitative Untersuchungen dieser Befunde sind im Gange.

\section{Experimentelles}

Sämtliche Arbeiten wurden unter strengstem Luftund Feuchtigkeitsausschluß durchgeführt.

3 E. O. Fischer u. D. Seus, Chem. Ber. 89, 1809 [1956].

4 F. Hein, P. Kleinert u. E. Kurras, Z. anorg. allg. Chem. 289, 229 [1957].

5 H. H. Zeiss u. W. Herwig, Liebigs Ann. Chem. 606, 209 [1957].
Darstellung von $\mathrm{Cr}\left(\mathrm{C}_{6} \mathrm{H}_{5}\right)_{3} \cdot 2 \mathrm{THF}$

Reines $\mathrm{Cr}\left(\mathrm{C}_{6} \mathrm{H}_{5}\right)_{3} \cdot 3$ THF wird mit dem entsprechenden Lösungsmittel versetzt und geschüttelt; nach erfolgter Umwandlung werden die grünen Kristalle abfiltriert und im Vakuum getrocknet.

Analyse:

Ber. für $\mathrm{Cr}\left(\mathrm{C}_{6} \mathrm{H}_{5}\right)_{3} \cdot 2 \mathrm{THF}$ :

Cr 12,17 C 73,1 H 7,3 ,

$\mathrm{C}_{6} \mathrm{H}_{5} \mathrm{HgCl}: \mathrm{Cr} 3: 1$ THF : Cr $2: 1$,

Gef.: (im Mittel)

Cr 12,1 C 74,2 H 7,15,

$\mathrm{C}_{6} \mathrm{H}_{5} \mathrm{HgCl}: \mathrm{Cr} 2,91: 1$ THF : Cr 2,1 : 1 .

Magnetische Messungen:

$$
\begin{aligned}
& \chi_{\mathrm{g}}=15,10 \cdot 10^{-6} \mathrm{~cm}^{3} / \mathrm{g} \text { bei } 291,2^{\circ} \mathrm{K} \\
& \chi_{\mathrm{g}}=14,98 \cdot 10^{-6} \mathrm{~cm}^{3} / \mathrm{g} \text { bei } 290,8^{\circ} \mathrm{K} . \\
& \text { Gewinnung des Bis-diphenylchrom }(O)
\end{aligned}
$$

Triphenylchromtristetrahydrofuranat wird mit Diäthyläther versetzt und einige Tage stehen gelassen. Nach dieser Zeit wird der Äther vorsichtig durch eine Kältedestillation entfernt und der Rückstand mit Hexan im Vakuum extrahiert, bis dieses praktisch farblos abläuft. Der Hexanextrakt wird wiederum durch Kältedestillation zur Trockne eingeengt und aus dem Rückstand das Diphenyl im Ölpumpenvakuum bei $40^{\circ} \mathrm{C}$ Badtemperatur absublimiert.

Diese Arbeit wurde unter der Leitung des Direktors der Forschungs. stelle für Komplexchemie der Deutschen Akademie der Wissenschaften zu Berlin, Jena, Herrn Prof. Dr. Dr. h. c. Fr. Hein durchgeführt. Für die Úberlassung des Themas, die Bereitstellung von Mitteln sowie sein ständig förderndes Interesse sei ihm an dieser Stelle gedankt. Danken möchten wir auch Herrn Dr. E. Kurras (Forschungsstelle für Komplexchemie) für seine anregenden Diskussionen, Herrn Dr. W. SEIDEL (Inst. f. Anorg. Chemie Jena) für die Ausführung der magnetischen Messunf. Anorg. Chemie Jena) für die Ausführung der magnetischen Messun-
gen und Herrn Dipl.-Chem. G. Marx (Inst. f. Physikal. Chem. Jena) für die Aufnahme und Diskussion der IR-Spektren.

6 T. F. Burger u. H. H. Zeiss, Chem. u. Ind. 1962, 183; M. Tsutsur, Ann. New York Acad. Sci. 93, 133 [1961]; M. Tsursui u. H. H. Zeiss, J. Amer. chem. Soc. 81, 1367 [1959].

* Die Isotopenanalyse wurde freundlicherweise vom Institut für stabile Isotope der Deutschen Akademie der Wissenschaften zu Berlin durchgeführt.

\section{$\boldsymbol{N}$-Dimethylmethioninol - eine Substanz mit Camembert-Aroma}

\author{
Von Th. Eckert, A. Knieps und H. Hoffmann
}

Pharmazeutisches Institut der Universität Frankfurt/Main (Z. Naturforschg. 19 b, 1082-1083 [1964]; eingeg. am 13. August 1964)

Während der Käsereifung entstehen durch Proteolyse zahlreiche freie Aminosäuren, die z. T. weiter biochemisch abgebaut werden. Als Aroma- und Duftstoffe der verschiedenen Käsesorten werden hauptsächlich Fettsäuren, Aminosäuren, Alkohole und Carbonylverbindungen angegeben ${ }^{1}$.
Über den speziellen Aromastoff des CamembertKäses, der von Penicillium Camemberti erzeugt wird, ist bisher nichts Näheres bekannt. Insbesondere die biochemischen Reduktionsprodukte des Methionins schienen uns in diesem Zusammenhang interessant. Das Methioninol (2-Amino-4-methylthio-butanol-1), das zunächst synthetisiert und auf seinen Geruch überprüft wurde, zeigte einen charakteristischen, aber nur wenig an Camembert-Käse erinnernden Geruch.

1 Zusammenfassend dargestellt: J. Schormüller, Lehrbuch der Lebensmittelchemie, Springer-Verlag, Berlin, Göttingen, Heidelberg 1961, S. 353. 